\title{
An Overview of High Performance Computing and Challenges for the Future
}

\author{
Jack Dongarra \\ University of Tennessee, USA
}

\begin{abstract}
In this talk we examine how high performance computing has changed over the last 10-year and look toward the future in terms of trends.

These changes have had and will continue to have a major impact on our software. A new generation of software libraries and algorithms are needed for the effective and reliable use of (wide area) dynamic, distributed and parallel environments.

Some of the software and algorithm challenges have already been encountered, such as management of communication and memory hierarchies through a combination of compile-time and run-time techniques, but the increased scale of computation, depth of memory hierarchies, range of latencies, and increased run-time environment variability will make these problems much harder.

We will focus on the redesign of software to fit multicore architectures.
\end{abstract}

